\title{
Visualizing Asymmetric Features of Icosahedral Viruses
}

\author{
Robert Ashley*, Jennifer Yoder, Susan Hafenstein \\ *Department of Microbiology and Immunology, The Pennsylvania State University College of \\ Medicine, 500 University Drive, Hershey Pennsylvania, 17033
}

The structure of virus and receptor interactions provides detailed information on attachment to host cells, which controls host range and largely dictates pathogenesis. Elucidating details of attachment can lead to a more complete understanding of the viral life cycle. Although many viruses are icosahedral when they initially bind to one or more receptor molecules on the cell surface, such an interaction is asymmetric, probably causing a breakdown in the symmetry and conformation of the original infecting virion in preparation for membrane penetration and release of the viral genome. Cryoelectron microscopy and biochemical analyses have shown that transferrin receptor (TfR), the cellular receptor for canine parvovirus can bind to only one or a few of the 60 icosahedrally-equivalent sites on the virion, indicating that either canine parvovirus has inherent asymmetry or binding of receptor induces asymmetry [1]. Asymmetric interactions of icosahedral viruses with their hosts might be a more common phenomenon than previously thought and may have been obscured by averaging in previous crystallographic and electron microscopic structure determinations.

To visualize the asymmetric interaction between virus and receptor, we have adapted a method used by Kelly et al $[2,3]$. His ${ }_{6}$-tagged TfR molecules were immobilized in a Ni-NTA head-group lipid monolayer that was formed directly on the freshly glow-discharged EM grid. Subsequently, the virus was applied to the TfR-studded grid thus allowing formation of virus-receptor complex directly on the EM grid. Furthermore, this approach more closely resembles a biological reality by presenting the receptor in a mock membrane to interact asymmetrically with the virus for visualization by single particle reconstruction methods. The presentation has the added advantage of optimizing the collection of thousands of complex particles necessary for high resolution asymmetric reconstructions. This technique is being used in other applications to illustrate the dynamics and asymmetry of small icosahedral viruses during the virus lifecycle.

[1] Hafenstein et al., Proc Natl Acad Sci U S A. 2007 Apr 17;104(16):6585-9

[2] Kelly et al., J Mol Biol. 2008 Oct 3; 382(2); 423-433

[3] Kelly et al., J Mol Biol. 2010 Jul 23;400(4):675-81

This research was supported by NIH 1 K22 AI 079271 and NIH-R01 AI 092571-01 